This document is the Accepted Manuscript version of a Published Work that appeared in final form in Chemistry of Materials, copyright (c) American Chemical Society after peer review and technical editing by the publisher. To access the final edited and published work see:

https: //dx.doi.org/10.1021/acs.chemmater.5b04406. 


\title{
Size-controlled Synthesis of sub-10 nm Citrate-stabilized Gold Nanoparticles and Related Optical Properties.
}

\author{
Jordi Piella ${ }^{\mathrm{a}, \mathrm{d}}$, Neus G. Bastús ${ }^{\mathrm{a}}$ and Victor Puntes $\mathrm{s}^{\mathrm{a}, \mathrm{b}, \mathrm{c}, *}$ \\ ${ }^{a}$ Institut Català de Nanociència i Nanotecnologia (ICN2), Campus UAB, 08193 Bellaterra, Barcelona, Spain. \\ ${ }^{\mathrm{b}}$ Vall d'Hebron Institut de Recerca (VHIR), 08035, Barcelona, Spain. \\ ${ }^{\mathrm{c}}$ Institució Catalana de Recerca i Estudis Avançats (ICREA), 08010 Barcelona, Spain. \\ ${ }^{\mathrm{d}}$ Universitat Autònoma de Barcelona (UAB), Campus UAB, 08183 Bellaterra, Barcelona, Spain.
}

* To whom correspondence should be addressed: E-mail: victor.puntes@icn.cat

\begin{abstract}
Highly-monodisperse biocompatible and functionalizable sub-10 $\mathrm{nm}$ citrate-stabilized gold nanoparticles (Au NPs) have been synthesized following a kinetically controlled seeded-growth strategy. The use of traces of tannic acid together with an excess of sodium citrate during nucleation is fundamental in the formation of a high number $\left(7 \cdot 10^{13} \mathrm{NP} / \mathrm{mL}\right)$ of small $\sim 3.5 \mathrm{~nm}$ seeds with a very narrow distribution. A homogeneous nanometric growth of these seeds is then achieved by adjusting the reaction parameters: $\mathrm{pH}$, temperature, sodium citrate concentration and gold precursor to seeds ratio. We use this method to produce Au NPs with a precise control over their sizes between 3.5 and $10 \mathrm{~nm}$ and a versatile surface chemistry allowing studying the size-dependent optical properties in this transition size regime lying between clusters and nanoparticles. Interestingly, an inflexion point is observed for NPs smaller than $8 \mathrm{~nm}$ in which the sensitivity of the Localized Surface Plasmon Resonance (LSPR) peak position as a function of NPs size and surface modifications dramatically increased. These studies are relevant in the design of the final selectivity, activity and compatibility of $\mathrm{Au}$ NPs, especially in those (bio)applications where size is a critical parameter (e.g. biodistribution, multiplex labeling and protein interaction).
\end{abstract}




\section{INTRODUCTION}

Gold nanoparticles (Au NPs) are still one of the most interesting nanomaterials due to their exceptional chemical stability, catalytic activity, processability and metallic nature, which provide them unique size-dependent optical and electronic properties. As a result, Au NPs are used in a wide variety of technologies including catalysis ${ }^{1}$, nanoelectronics ${ }^{2}$ and biomedicine ${ }^{3}$ (sensing, diagnosis, imaging and labeling). In order to develop nanomaterials to be suitable for the "bottom-up" design of their applicability, their synthesis must lead to monodispersed entities with structure-controlled features that exhibit reproducible properties. ${ }^{4}$ Among all sizes, highly monodisperse and functionalizable sub-10 $\mathrm{nm}$ Au NPs with tunable surfaces are appealing materials. In this regime, the percentage of surface atoms starts taking over bulk atoms, and their atomic coordination at the surface decreases, increasing their catalytic properties and resulting in large variations in the particles responses and reactivity (i.e. plasmon shifts, corrosion, dissolution, stability...). ${ }^{5-8}$ Besides, these $\mathrm{Au}$ NPs are especially attractive for biomedical applications and nanomedicine since many body barriers falls in the sub-10 $\mathrm{nm}$ regime therefore determining NP's biodistribution, accumulation in tissues, excretion and interaction with proteins. ${ }^{9-12}$

Most common strategies for the synthesis of sub-10 nm Au NPs are based on the use of strong reducing agents, i.e. sodium borohydride, in the presence of strong capping ligands that quench $\mathrm{Au}$ NP's growth, following either a two-phase liquid-liquid method (the so-called Brust method ${ }^{13}$ ) or single-phase approaches. This is the case of small hydrophobic gold clusters coated by phosphines $(1.4-1.5 \mathrm{~nm})^{14,15}$, amines $(2.5-7 \mathrm{~nm})^{16}$ and alkanethiols $(1-6 \mathrm{~nm}){ }^{13,17,18}$ Similarly, the use of hydrophilic ligands such as poly(ethylene glycol) $(\mathrm{PEG})(1.5-18 \mathrm{~nm})^{19-21}$, polymeric stabilizers (1.5-4 $\mathrm{nm})^{22}$, strong binding cationic surfactants $(4-6 \mathrm{~nm})^{23,24}$ and other thiol-functionalized molecules $(0.7-$ $3.2 \mathrm{~nm})^{25,} 26$ allow producing Au NPs in this small size regime. ${ }^{27-29}$ However, at the end of the synthesis, their surfaces are passivated by a strong binding surfactant layer. In the particular case of aqueous syntheses, it is especially interesting the well-established protocol producing Au NPs in the range 5-40 $\mathrm{nm}$ based on the use of cetyltrimethylammonium bromide (CTAB), initially reported by Murphy and co-workers ${ }^{30,31}$. Here, as in the case of the syntheses in organic solvents, and despite the 
excellent size control provided by this method, the use of non-biocompatible reagents, which entail tedious "detoxification" steps, and/or the use of strong surfactants that blocks NP's surface limiting further functionalization, restricts their final use. ${ }^{32}$ In this context, the question is still how to produce $\mathrm{Au}$ NPs in the small regime, without restricting surface accessibility, while gaining reproducible control over particles' size distribution.

One of the most popular synthetic methods for the production of Au NPs is the aqueous reduction of tetrachloroauric acid $\left(\mathrm{HAuCl}_{4}\right)$ by sodium citrate, pioneered by Turkevich ${ }^{33}$ and further refined by Frens. ${ }^{34}$ The interest of this method relies on the resulting versatile citrate layer on the NP's surface which allows an easy (multi-)functionalization of the resultant particles with almost any type of molecules ${ }^{35,36}$ (including peptides, proteins, and DNA). Many protocols have been developed for the preparation of citrate-stabilized Au NPs, leading to the production of fairly monodisperse quasispherical particles from about 10 to $300 \mathrm{~nm}$ in diameter by simply varying the reaction parameters (solution $\mathrm{pH}^{37,38}$, reducing agents ${ }^{39-44}$, solvent $\mathrm{t}^{38,45}$ and order of reagents addition ${ }^{46,47}$ ). Later, seededgrowth based methods resulted in improved size distributions, leading to highly monodisperse samples. $^{30,38,48,49}$ However, this strategy has not yet been adapted to produce Au NPs below $10 \mathrm{~nm}$, therefore limiting the availability of high quality samples in this small size regime.

Herein, we take advantage of the recent improvements on the kinetic control of seeded-growth synthesis of citrate-stabilized metal $\mathrm{NPs}^{49,50}$ to produce Au NPs with controlled morphology and nanometric size resolution between 3.5 and $10 \mathrm{~nm}$. Controlled nucleation of $3.5 \mathrm{~nm} \mathrm{Au} \mathrm{NPs} \mathrm{is}$ achieved by the combined use of two competing reducing agents: tannic acid and sodium citrate. The use of these two reducing agents for the synthesis of Au NPs was initially introduced in the seminal work of Mühpfordt in 1982 and further refined by Slot and Geuze in 1985 as an effective mean to produce NPs smaller than those obtained in the standard Turkevich method. ${ }^{51-53}$ In this work, we develop a seeded-growth method that allows producing highly monodisperse $3.5 \mathrm{~nm}$ Au NP's seeds that can be grown, with nanometric resolution, up to the desired size, and accessible surfaces. While the synthesis of the seed particles involves the combined use of both reducers, the growth process is controlled exclusively by the use of sodium citrate and the fine adjustment of synthetic parameters 
(temperature, $\mathrm{pH}$, seeds concentrations and $\mathrm{SC}$ to gold precursor ratio). Thus, the initial nucleation promoted by one of the reducers determines the final number of NPs, while the growth of those initial seeds mediated by the other reducer determines their final size. As a result, stable quasi-spherical and functionalizable highly monodisperse smaller than $10 \mathrm{~nm}$ Au NPs have been produced.

Furthermore, due to their narrow size distributions and versatile surface chemistry, as-synthesized $\mathrm{Au}$ NPs can be used as a model material to study the unique size-dependent optical properties in this small size regime. This regime, lying in the frontier between clusters and large NPs, is especially interesting as size and surface chemistry effects become increasingly important.

\section{RESULTS AND DISCUSSION}

\section{Synthesis of $3.5 \mathrm{~nm}$ seeds Au NPs}

The addition of traces of tannic acid (TA) to the Inverse Turkevich method ${ }^{49}$ substantially modifies the kinetics of Au NP's formation, leading to the fast and systematic production of narrowly dispersed $3.5 \mathrm{~nm} \mathrm{Au}$ NPs (Figure 1). Briefly, the Au NPs were obtained by injecting $1 \mathrm{~mL}$ of tetrachloroauric acid $\left(\mathrm{HAuCl}_{4}, 25 \mathrm{mM}\right)$ into a mixed solution containing $150 \mathrm{~mL}$ of sodium citrate $(\mathrm{SC}, 2.2 \mathrm{mM})$ and $0.1 \mathrm{~mL}$ of tannic acid (TA, $2.5 \mathrm{mM}$ ) at $70^{\circ} \mathrm{C}$. Thus, the solution turned from transparent to dark grey instantaneously after gold precursor injection, and then to brownish-orange within a few minutes, indicative of the formation of very small Au NPs. The use of traces of TA, with stronger reducing power than SC, appeared to be crucial for the formation of these small particles. However, both reducing agents were needed, otherwise the resultant particles presented large and rather polydisperse sizes (Figure 1A). The absorption profile obtained when both reducers were combined (Figure 1B) reveals a damped Localized Surface Plasmon Resonance (LSPR) band peaking around 504-505 nm, which is in accordance of very small Au NPs. ${ }^{54}$ Similarly, absorption profiles centred at $518 \mathrm{~nm}$ and $527 \mathrm{~nm}$ obtained when using only SC or TA, respectively, correlate well with the larger sizes seen by TEM. These results further indicate the synergic effect resulting from the combination of the two 
reducers. HR-TEM morphological characterization shows the faceted (twinned) nature of the synthetized particles along with its high monodispersity (Figure 1C and 1D).
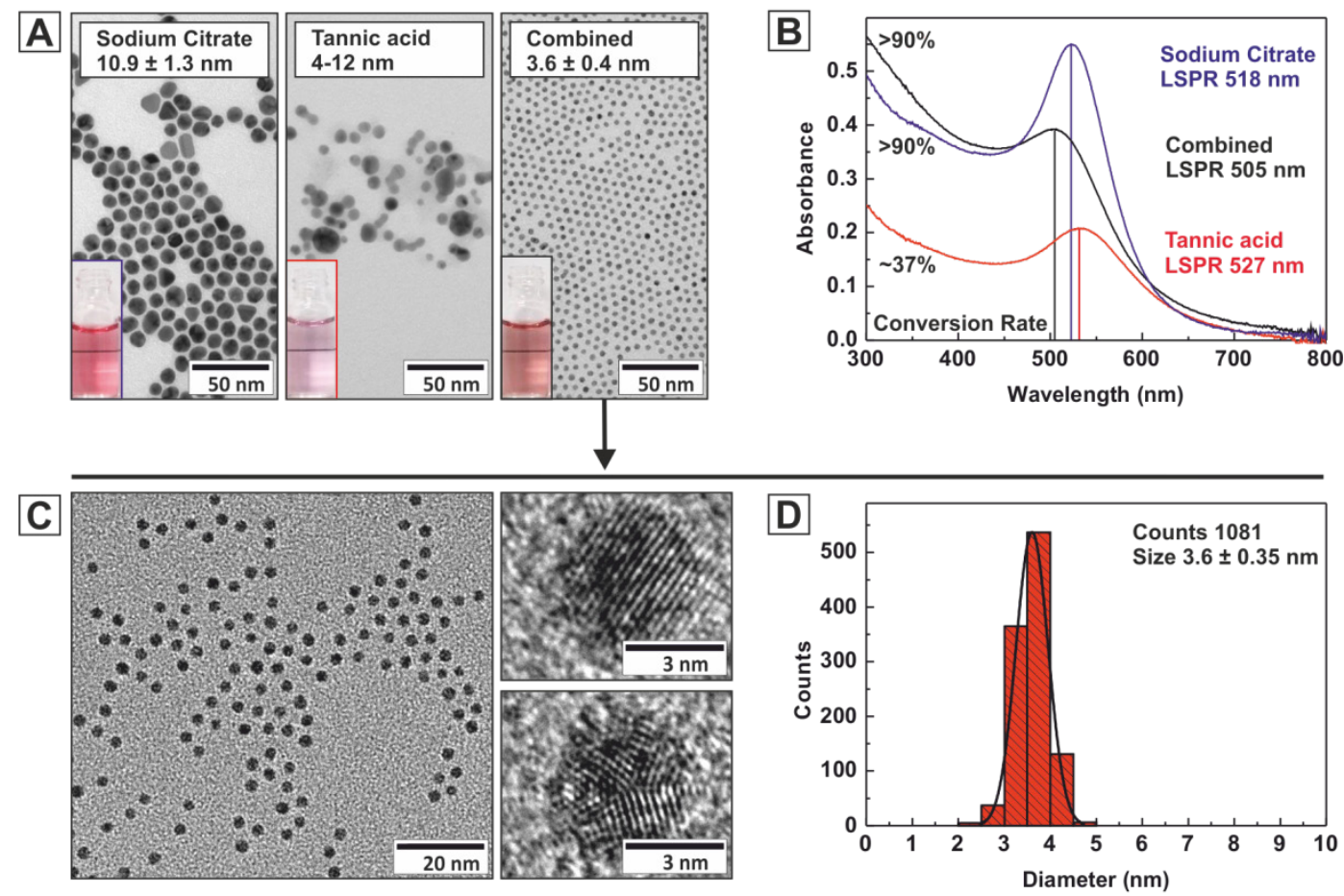

Figure 1. (A) Representative TEM images of $\mathrm{Au}$ NPs synthesized by injecting $1 \mathrm{~mL}$ of $\mathrm{HAuCl}_{4}(25$ $\mathrm{mM}$ ) to a $150 \mathrm{~mL}$ solution of: SC, TA or the combination of both SC and TA. Refer to Supporting Information Section 2.1 for experimental details. (B) Corresponding UV-Vis spectra and estimated precursor conversion rate, by measuring the absorbance at $400 \mathrm{~nm}^{55}$ of the Au colloidal solutions obtained in A. (C) High resolution TEM images of $\sim 3.5 \mathrm{~nm}$ Au NPs with individual particle image indicating multiple-twin boundaries. (D) Size distribution profile showing the highly monodispersity of the sample.

\section{Effect of Reaction Parameters on the Synthesis of seeds Au NPs}

In order to obtain the smallest Au NPs with the narrowest size distributions, three different synthetic parameters play a critical role: $\mathrm{pH}, \mathrm{TA}$ concentration and temperature. It is well established that reaction $\mathrm{pH}$ defines the reactivity of the gold precursor and the protonation state of the citrate ions, finally determining the reaction mechanism, and therefore the final Au NP morphology. ${ }^{38}$ On the other hand, the kinetics of reduction reaction is mainly controlled by the concentration of TA and the temperature, which governs the degree of monomer supersaturation, ultimately regulating the number and size of nuclei. 
The effect of these parameters, that is the $\mathrm{pH}$, concentration of TA and temperature, was studied and are summarized in Figure 2. Effect of the $p H$. As seen in Figure 2A, the final morphology of the $\mathrm{Au}$ NPs is strongly determined by the $\mathrm{pH}$ of the reaction mixture. The optimal size control was achieved at slightly basic $\mathrm{pH}(\mathrm{pH} \sim 8)$, by correcting the reaction mixture with $\mathrm{K}_{2} \mathrm{CO}_{3}$, leading to the production of quasi-spherical Au NPs with small sizes and narrower distributions. On the contrary, the formation of anisotropic shapes and/or larger particles was observed at rather acidic or alkaline conditions, respectively. These results can be explained in terms of both the protonation of citrate ions and the strong $\mathrm{pH}$-dependent reduction potential of $\mathrm{Au}(\mathrm{III})$ complexes. Thus, at acidic values the protonation of citrate $\left(\mathrm{pK}_{1} 3.1, \mathrm{pK}_{2} 4.6\right.$ and $\left.\mathrm{pK}_{3} 6.4\right)$ results in the poor stability of the particles while at alkaline values $\mathrm{Au}$ ions become less reactive due to the formation of stable hydroxide complexes. ${ }^{38}$ As a result, the optimal $\mathrm{pH}$ value is found to be that which balances both the reactivity of the gold precursor to promote a fast nucleation while ensuring a good electrostatic stabilization of the particles. Effect of the $T A$. Beyond the optimization of the $\mathrm{pH}$, the kinetics of the reaction was highly dependent on the TA concentration (Figure 2B). As a general trend, an initial increase of the concentration of TA increased the reaction kinetics, which was translated into smaller sizes, down to $3.5 \mathrm{~nm}$. However, a further excess of TA (see Supporting information Section 1 for stoichiometry) resulted in polydisperse samples. The reason is still not fully understood but the presence of very small clusters (sub $3 \mathrm{~nm}$ ) together with a high number of larger particles in these samples indicate some lack of stability of the resultant NPs, presumably due to the saturation of the nucleation process and the consequent rapid nucleation and aggregative growth, together with coalescence, of these small nuclei. Thus, the limiting factor for achieving the smallest Au NP's size is not determined by the reducing power of the TA but by the ability to stabilize the NPs. Effect of the temperature. Similarly, formation kinetics of the Au NPs was also dependent on the reaction temperature (Figure 2C). At low temperatures (below $70{ }^{\circ} \mathrm{C}$ ) the growth process is favoured, which was translated into larger Au NPs but preserving highly monodisperse sizes. Oppositely, the sample started to become smaller and more polydisperse when the temperature was increased (above $70{ }^{\circ} \mathrm{C}$ ) and the size decreased below $3.5 \mathrm{~nm}$, in a similar trend to that observed in the case of the TA concentration. Unfortunately, any attempt to decrease the size 
of the Au NPs below $3.5 \mathrm{~nm}$ by further modifying these synthetic parameters resulted in polydisperse samples. Although some hypothesis has been introduced here, this is a subject of ongoing research.

From these results it can be concluded that the optimal conditions for the production of high quality colloidal solutions of quasi-spherical Au NPs with the smallest possible size involves: (i) the adjustment of the reaction $\mathrm{pH}$ at slightly basic values $(\mathrm{pH} \sim 8)$ (ii) the use of sub-stoichiometric concentration of TA instead of an excess of that one and (iii) moderately high temperatures $\left(\sim 70^{\circ} \mathrm{C}\right)$. Working at these conditions long-term stable Au colloids of $\sim 3.5 \mathrm{~nm}$ and standard deviation less than $10 \%$ were routinely produced. Interestingly, under these conditions the synthetic method was easily scaled up to 1 L (Figure S1).

Generally, the observed trend fits well with a mechanism of NP's formation divided in two steps: a nucleation promoted by a strong reducer, TA, and particle growth promoted by a weak reducer, SC. When TA is used under sub-stoichiometric conditions, the rate of nucleation and the final size of NPs are determined by the conversion rates of both processes. Thus, TA leads to the production of initial nuclei that are subsequently grown by the further reduction of the remaining gold precursor by the SC present in the medium. This strategy of competing reducers has been previously exploited in the control of Au NP size ${ }^{53}$ and the morphology of Pt NPs in organic solvents. ${ }^{56,57}$ When increasing the TA concentration, more gold is reduced during the nucleation and less by SC in the post-nucleation process, which leads to a decrease in the Au NP's size while preserving a narrow distribution. Considering that the Au conversion from precursor to NP yield is the same in all cases since an excess of SC is always used (about $>90 \%$ ), the number and size of NPs is then coupled (from many small NPs to fewer larger ones). Interestingly, a similar behaviour is observed when modifying the temperature of the reaction. 

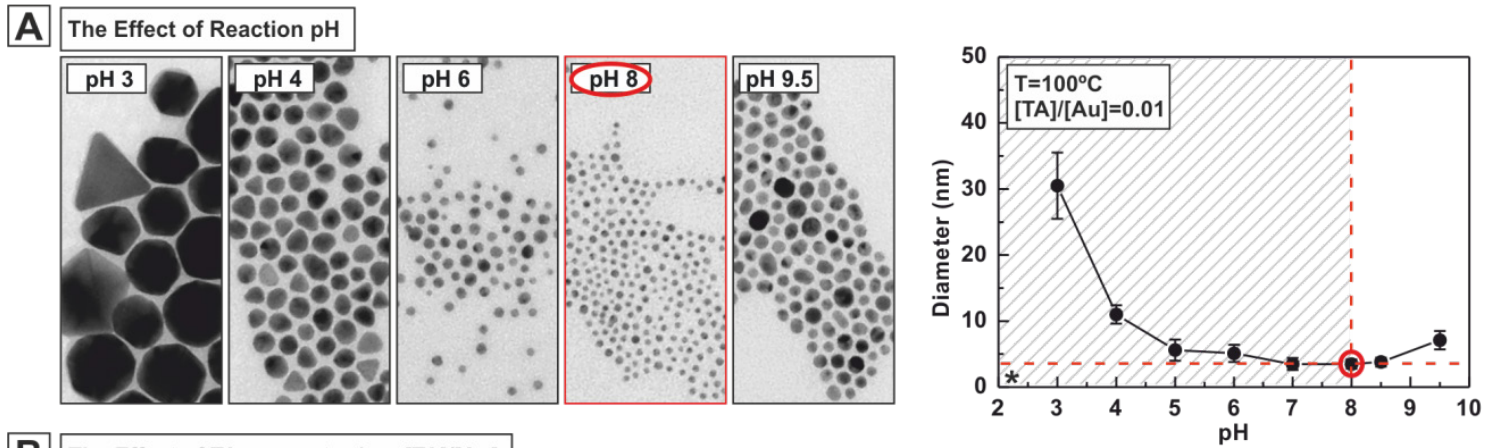

B The Effect of TA concentration, [TA][/Au]
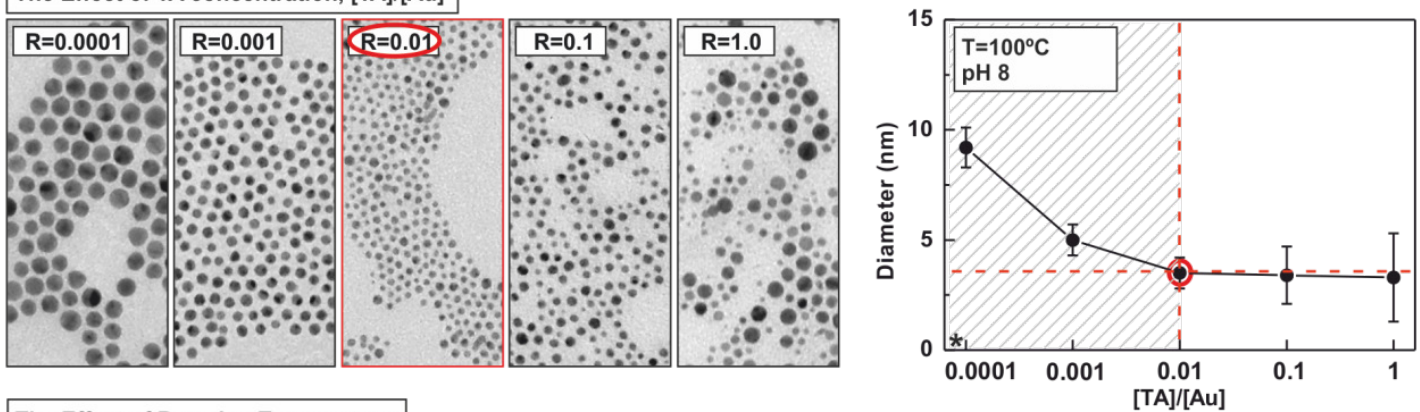

C The Effect of Reaction Temperature
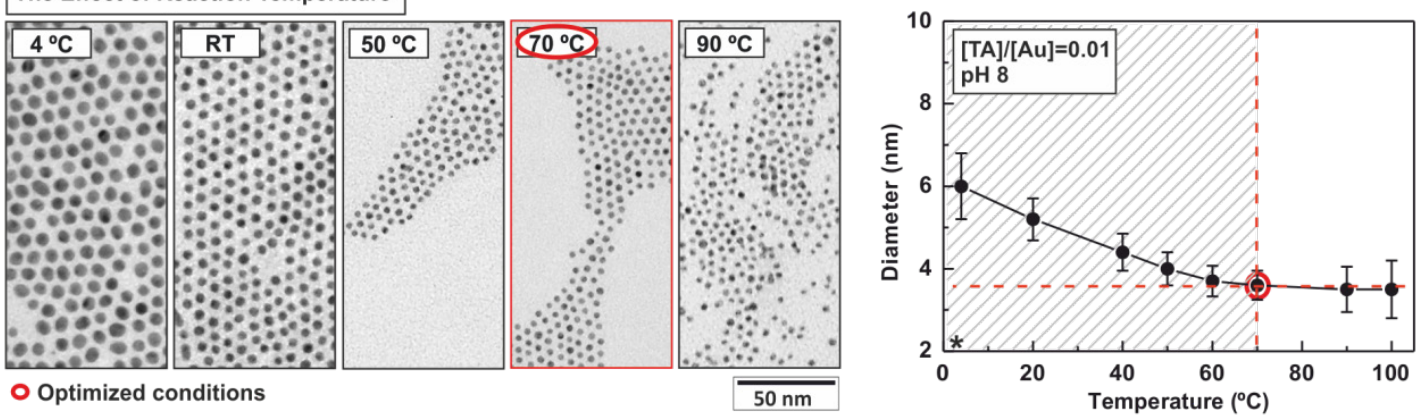

Figure 2. Different tested conditions for the nucleation of Au NPs. In all cases $1 \mathrm{~mL}$ of $\mathrm{HAuCl}_{4}(25$ $\mathrm{mM})$ was injected to a $150 \mathrm{~mL}$ solution of $\mathrm{SC}(2.2 \mathrm{mM})$ and TA. Citric acid and $\mathrm{K}_{2} \mathrm{CO}_{3}$ was used to adjust the $\mathrm{pH}$. Refer to Section 2.2 of the Supporting Information for detailed conditions. (A) Evaluation of the effect of reaction $\mathrm{pH}$. Reaction $\mathrm{pH}$ was systematically varied while all other synthetic parameters were kept constant. (B) Evaluation of the effect of TA. The amount of TA was varied while all other synthetic parameters were kept constant. (C) Evaluation of the effect of reaction temperature. The temperature of the reaction was modified while all other synthetic parameters were kept constant. *Error bars are indicative of the size standard distribution.

\section{Seeded-growth Synthesis of Au NPs from $3.5 \mathrm{~nm}$ to $10 \mathrm{~nm}$}

Given the limitations in controlling Au NP's size by modifying the concentration of TA, temperature or $\mathrm{pH}$ we studied the possibility of growing successive generations of spherical Au NPs using the smallest $\sim 3.5 \mathrm{~nm}$ above synthesized Au NPs as seeds. Taking advantage of the recent improvements on controlled growth of $\mathrm{Au}^{49}$ and $\mathrm{Ag}^{50} \mathrm{NPs}$ from $10 \mathrm{~nm}$ up to $200 \mathrm{~nm}$, we set the conditions by which 
the kinetic control of the seeded-growth synthesis is achieved, that is, (i) slowing down the reactivity of gold precursor to avoid new nucleation while (ii) maintaining its concentration high enough to achieve an homogeneous growth (size focusing). These conditions include the control of the reaction temperature, $\mathrm{pH}$ and precursor to seed particles ratio.

Thus, in order to obtain successive generations of highly monodisperse Au NPs up to $10 \mathrm{~nm}$, Au seeds $\left(3.5,7 \cdot 10^{13} \mathrm{NPs} / \mathrm{mL}\right)$ were first prepared as previously described. When the reaction was finished the solution was diluted by extracting a sample volume and replacing it with the same volume of $2.2 \mathrm{mM}$ of SC solution (see Scheme S2) in order to reduce the number of growing seeds while ensure enough $\mathrm{SC}$ for subsequent gold reduction and $\mathrm{pH}$ buffering. During the growth process, the temperature was set at $70{ }^{\circ} \mathrm{C}$ and no TA was added. At this temperature growth is promoted over nucleation by the weak reducing power of SC. Finally, subsequent aliquots of gold precursor were injected. By repeating this process (dilution plus gold precursor injection) the size of the particles was precisely controlled with an increase of approximately $0.5-1 \mathrm{~nm}$ after each injection.

Morphological characterization by TEM of the resultant generations of Au NPs obtained after different growth steps are summarized in Table $\mathbf{1}$ and shown in Figure 3. Au NPs, progressively larger in size, exhibit high uniformity and very low size dispersity. Size distribution studies performed by the systematical analysis of at least 500 particles for each sample further corroborate the results (Figure S2). Similar size distribution profiles were measured by dynamic light scattering (DLS), obtaining monomodal curves with increasing mean particles diameter (Figure 4A). Besides, particle's size can be also estimated considering the diameter of the seeds, its number in solution (concentration) and the amount of gold precursor added (refer to Supporting Information Section 3.2). Experimental results (TEM, DLS) fits well with those theoretically calculated (Figure 4B), obtaining a perfect correlation and further confirming both the high reaction yield and the absence of new nucleation. ${ }^{49}$ Finally, an extension of the growth process up to $20 \mathrm{~nm}$ is shown in Section 3.4 of the Supporting Information. 
Table 1. Summary of Au NP's size and concentration obtained after different growth steps.

\begin{tabular}{cccccc}
\hline $\begin{array}{c}\text { Precursor } \\
\text { Injections }\end{array}$ & $\begin{array}{c}\text { TEM } \\
\text { Diameter (nm) }\end{array}$ & SD (\%) & $\begin{array}{c}\text { DLS } \\
\text { Diameter }(\mathbf{n m})^{\mathbf{a}}\end{array}$ & $\begin{array}{c}\text { Concentration } \\
(\mathbf{N P s} / \mathbf{m L})^{\mathbf{b}}\end{array}$ & $\begin{array}{c}\text { Expected Diameter } \\
(\mathbf{n m})^{\mathbf{b}}\end{array}$ \\
\hline Seeds & $3.6 \pm 0.4$ & 11.1 & $4.4 \pm 0.3$ & $7.0 \cdot 10^{13}$ & 4.4 \\
1 & $4.5 \pm 0.4$ & 8.9 & $4.8 \pm 0.3$ & $4.4 \cdot 10^{13}$ & 4.9 \\
2 & $5.1 \pm 0.5$ & 9.8 & $5.7 \pm 0.2$ & $4.4 \cdot 10^{13}$ & 5.6 \\
3 & $6.1 \pm 0.6$ & 9.8 & $6.5 \pm 0.2$ & $2.8 \cdot 10^{13}$ & 6.2 \\
4 & $6.6 \pm 0.6$ & 9.1 & $6.7 \pm 0.2$ & $2.8 \cdot 10^{13}$ & 6.9 \\
5 & $7.4 \pm 0.7$ & 9.5 & $7.7 \pm 0.4$ & $1.8 \cdot 10^{13}$ & 7.9 \\
6 & $7.9 \pm 0.7$ & 8.9 & $8.2 \pm 0.2$ & $1.8 \cdot 10^{13}$ & 8.3 \\
7 & $8.7 \pm 0.8$ & 9.2 & $9.1 \pm 0.1$ & $1.2 \cdot 10^{13}$ & 8.9 \\
8 & $9.5 \pm 0.8$ & 8.4 & $9.6 \pm 0.1$ & $1.2 \cdot 10^{13}$ & 9.8 \\
10 & $10.5 \pm 0.9$ & 8.6 & $10.1 \pm 0.4$ & $7.1 \cdot 10^{12}$ & 10.5 \\
\hline
\end{tabular}

${ }^{a}$ Number mean and SD from three independent runs. ${ }^{b}$ see Supporting Information Section 3.2 for theoretical calculations.

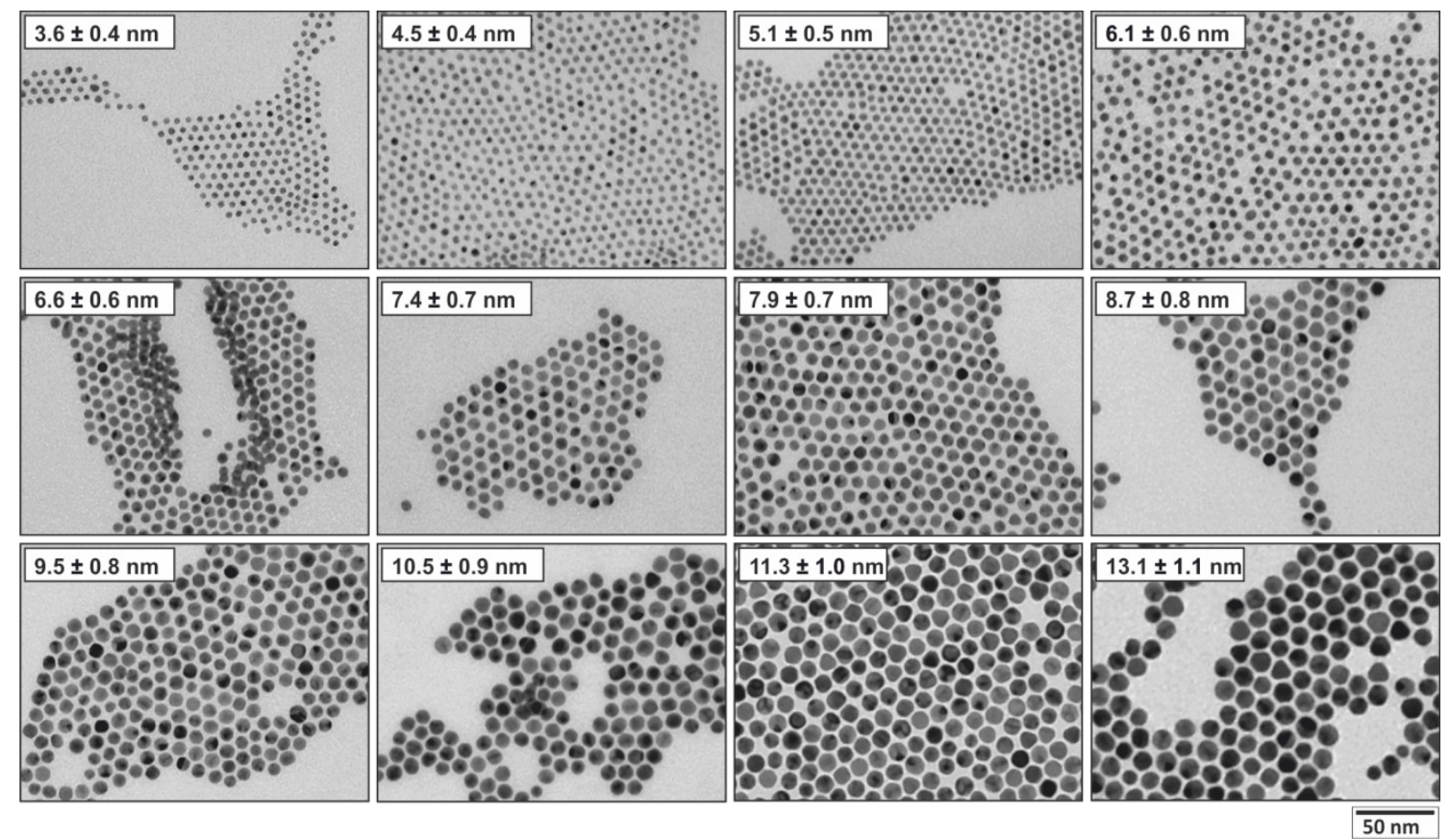

Figure 3. Transmission electron microscopy images of seed particles $(3.6 \mathrm{~nm})$ and those obtained after different growth steps. The particle size increases from $3.6 \pm 0.4 \mathrm{~nm}$ seeds to $13.1 \pm 1.1 \mathrm{~nm}$ while its concentration decrease from $\sim 7.0 \times 10^{13}$ to $\sim 4.5 \times 10^{12} \mathrm{NPs} / \mathrm{mL}$. 

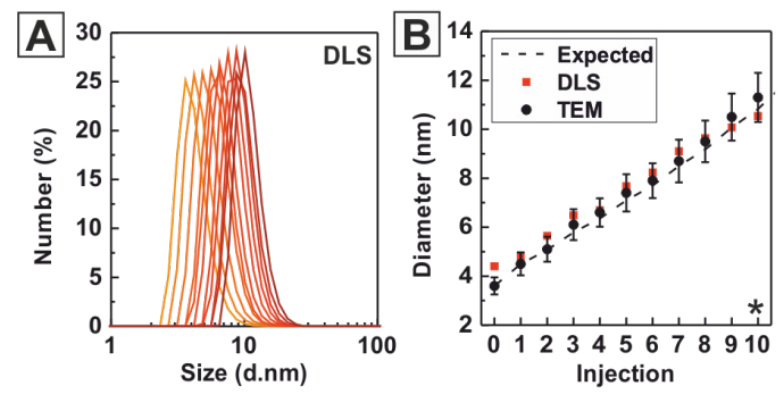

Figure 4. (A) Size distribution profiles measured by dynamic light scattering of Au NPs obtained after different growth steps. Size distribution by Number fits well with the size measured by TEM and shows monomodal curves with increasing mean particle diameters as the number of growth steps increase. Size distribution by Intensity and Volume are shown in Figure S3. (B) Experimental average diameter of Au NPs (TEM and DLS) obtained after several growth steps and comparison with the theoretical calculated values. Obtained results fit with those calculated, which indicates that no nucleation occurred and the Au atoms deposited onto the surfaces of the preformed particles. *Errors bar indicates the size standard distribution.

\section{Optical properties of Au NPs}

The unique optical properties of Au NPs are well known and enabling for many applications. Unfortunately, it is not always easy to produce Au NPs in a wide size range using the same procedure, in particular for the small ones. Taking advantage of the size resolution herein achieved, we measured the absorbance spectra of the different particles by UV-Vis spectroscopy (Figure 5), where it can be clearly seen how the Localized Surface Plasmon Resonance (LSPR) band narrows and the peak position red-shifts from $505 \mathrm{~nm}$ to $517 \mathrm{~nm}$ as increasing particle size from 3.6 to $11.3 \mathrm{~nm}$. Neither broadening of the band nor the appearance of new peaks at longer wavelengths was observed, indicating the absence of any aggregation. Two regions with different behavior are identified. Beyond the damping of the absorption band, for very small Au NPs $(<8 \mathrm{~nm})$, slight variations in the size (from $3.6 \mathrm{~nm}$ to $8 \mathrm{~nm}$ ) are translated into larger shifts in the LSPR peak position (from $505 \mathrm{~nm}$ to 515 $\mathrm{nm}$, respectively). For larger sizes $(>8 \mathrm{~nm})$, this size-dependence is smoother, leading to the smaller standard red-shifts observed in Au NPs (from $515 \mathrm{~nm}$ to $517 \mathrm{~nm}$ ) for similar size variations (from 8 $\mathrm{nm}$ to $13 \mathrm{~nm}$ ). Oppositely to that recently observed for sub-10 nm Ag NPs ${ }^{58}$ we did not detect a reverse tendency in the size-dependence of the LSPR, that is, for Au NPs, the peak gradually redshifts as the mean particle size increases. 
The higher susceptibility (measured in terms of LSPR shift) observed in this small size regime can be attributed to surface phenomena, that become pronounced in very small particles with a high percentage of surface atoms, and that ultimately account for intrinsic size effect corrections in the standard Mie theory. ${ }^{79}$ In particular, size-dependent modifications of the dielectric constant with respect to the bulk values due to surface damping. ${ }^{60}$ Thus, while red-shifts in large Au NPs are explained in terms of retardations effects (as well as to increasing contributions from multipolar terms), for smaller Au NPs these extrinsic size-effects become negligible and intrinsic size effects prevails, resulting in a the damping of the LSPR band with decreasing sizes and into additional shifts of the plasmon peak as herein observed. Obtained results are complementary to those experimentally suggested, ${ }^{21,54,59}$ but the improved resolution herein accomplished leads to the identification of nonnegligible alterations in the LSPR, clarifying the size dependent LSPR peak position for small Au NPs. Although the optical modeling of these systems is out of the scope of this paper, these results could be useful as a reference tool for testing theoretical models in the small size regime, basically based on quantum mechanics calculations, and traditionally difficult to model for being in the limit of Mie theory approximations. 

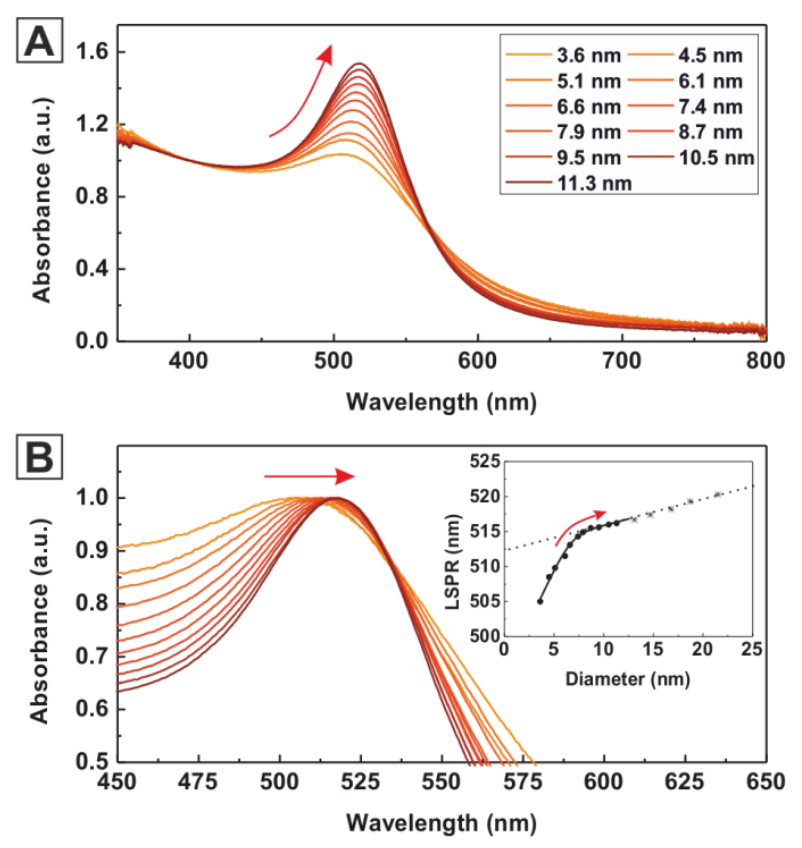

Figure 5. Optical properties of selected $\mathrm{Au}$ colloids obtained after different growth steps (A) normalized at $400 \mathrm{~nm}$ and (B) normalized at the LSPR peak maximum. Arrows indicate evolution of the LSPR peak as increasing the size of the Au NPs. In the inset graph, the position of the LSPR peak as a function of particle size is plotted, and extended up to particles of $20 \mathrm{~nm}$ for better understanding the tendency, where it can be distinguish: (i) the position of the peak always red shift as increasing the size of the particles and (ii) two size-regimes intersecting at $8 \mathrm{~nm}$ having different behavior.

\section{Functionalization of Au NPs}

Beyond the possibility to precisely control the size of Au NPs in the sub-10 nm regime, another advantage of this synthetic strategy is that it produces Au NPs with readily accessible and reactive surfaces. The weak interaction between Au surface and the carboxylic terminated groups of the citrate allows easy post-synthetic treatments, and the further functionalization of these Au NPs with a wide variety of (bio)molecules of interest. In order to proof this point we carried out conjugation experiments with 11-mercaptoundecanoic acid (MUA), a model thiol-terminated molecule widely used to build self-assembled monolayers (SAMs) onto Au NPs. Conjugation was performed by adding excess of MUA molecules to Au NPs solutions under vigorous stirring. Molecule adsorption is a spontaneous process because the thiol groups form much stronger bonds with $\mathrm{Au}$ surfaces than the carboxylic groups in the original citrate molecules do. ${ }^{61}$ Characterization of the Au NPs before and after MUA addition shows that neither aggregation nor observable structural or morphological change 
took place, confirming that the observed shift in the LSPR peak position is due to modifications on Au NP's surface chemistry. This shift could not be observed if the surface of the particles would be passivated by a strongly binding surfactant. Similarly to that previously observed, ${ }^{54,62}$ the presence of traces of TA used during the seed nucleation seems not to have a noticeable hindrance on surface accessibility.

Absorption spectra of the Au NPs before and after the conjugation process are summarized in Table 2 and shown in Figure 6 (extended up to $20 \mathrm{~nm}$, see Supporting Information Section 4.1) where it can be clearly seen how the corresponding LSPR peak position shifts to the red. Interestingly, the extent of the red-shift depends on Au NP size, being larger for smaller Au NPs (Figure 6 A-C). These results coincide with that observed recently for $\mathrm{Ag}_{\mathrm{NPs}}{ }^{58}$ of similar small sizes and arise from the higher fraction of surface atoms in smaller NPs which it is translated into the fact that similar chemically induced reduction of conduction band electrons (thiols confine more conduction band electrons than native carboxylic acids) produces a sharper effect. Therefore, in addition to the surface to volume ratio effect we clearly see, as in the previous section, higher susceptibility for the smaller particles.

The synthetic strategy herein presented allows to extend the previously citrate-mediated seededgrowth method for the production of Au NPs in the range of $10-200 \mathrm{~nm}^{49}$ towards smaller 3.5-10 nm sizes, covering the whole nanometric range. As a result we can observe, for the first time, how the susceptibility of the LSPR towards surface modifications (via ligand exchange processes) decreases as the size of the NP increases reaching a minimum around $50 \mathrm{~nm}$ before rising again as the NPs become larger, suggesting that around $50 \mathrm{~nm}$, surface and bulk plasmonic effects intersects (Figure 7). 
Table 2. Summary of the LSPR peak position of Au NPs before and after conjugation with 11mercaptoundecanoic acid (MUA).

\begin{tabular}{cccc}
\hline Size (TEM) & SPR $(\mathbf{n m})$ & SPR after functionalization $(\mathbf{n m}) *$ & $\lambda_{\text {SPR }}$ \\
\hline $3.6 \pm 0.4$ & 505.5 & 517.4 & 11.9 \\
$4.5 \pm 0.4$ & 508.0 & 518.5 & 10.5 \\
$5.1 \pm 0.5$ & 509.8 & 519.1 & 9.3 \\
$6.1 \pm 0.6$ & 511.8 & 520.5 & 8.7 \\
$6.6 \pm 0.6$ & 513.1 & 521.1 & 8.0 \\
$7.4 \pm 0.7$ & 514.5 & 522.0 & 7.5 \\
$7.9 \pm 0.7$ & 515.3 & 522.0 & 6.7 \\
$8.7 \pm 0.8$ & 516.3 & 522.8 & 6.5 \\
$9.5 \pm 0.8$ & 516.6 & 522.6 & 6.0 \\
$10.5 \pm 0.9$ & 517.2 & 523.0 & 5.8 \\
$11.3 \pm 1.0$ & 517.5 & 522.7 & 5.2 \\
$13.1 \pm 1.1$ & 518.0 & 522.9 & 4.9
\end{tabular}

*Au NPs were conjugated with 11-mercaptoundecanoic acid (MUA).
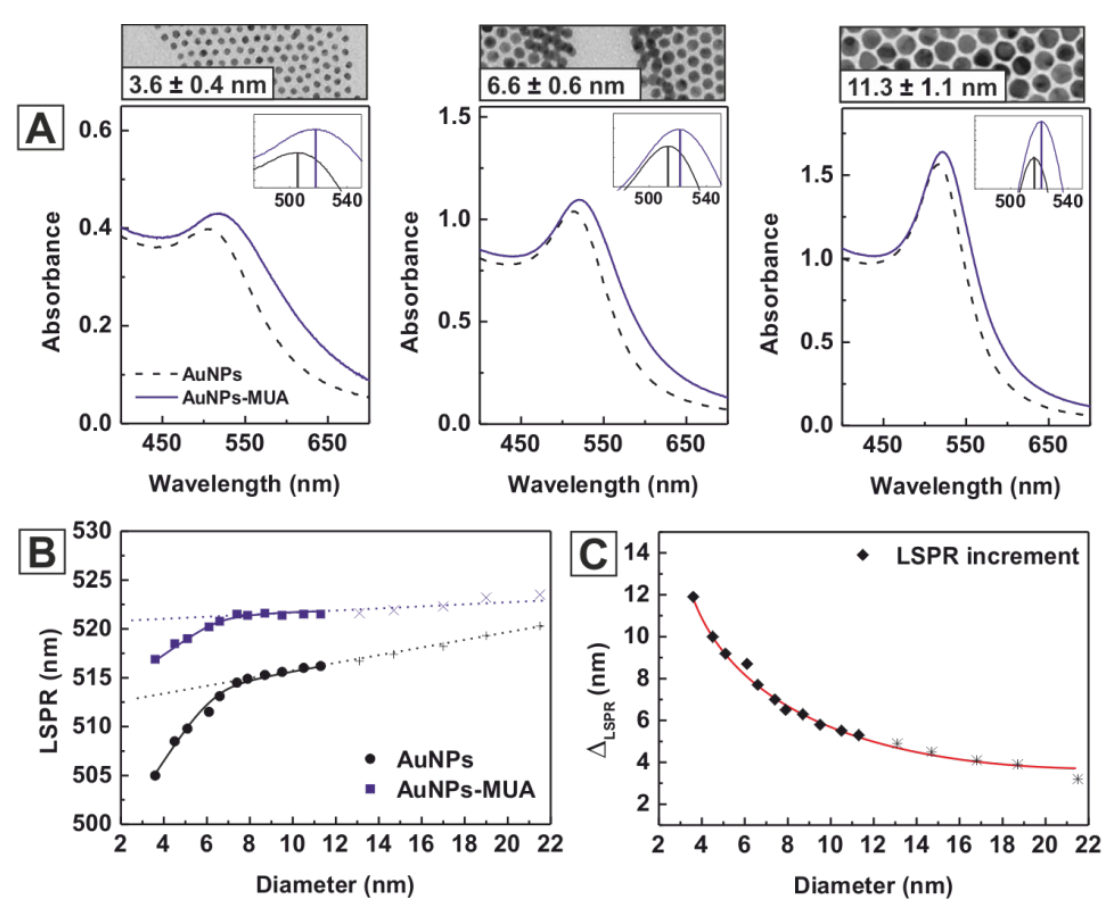

Figure 6. Conjugation of as-synthesized Au NPs with 11-mercaptoundecanoic acid (MUA).The size has been extended up to $20 \mathrm{~nm}$ for better understanding the tendency. (A) UV-vis spectra for different particle size: $3.6 \pm 0.4 \mathrm{~nm}, 6.6 \pm 0.58 \mathrm{~nm}$, and $11.3 \pm 1.0 \mathrm{~nm}$, recorded before (black dotted line) and after (red line) the addition of MUA. Lines indicate the position of the LSPR peak. (B) Sizedependence evolution of the LSPR peak position as a function of Au NP size before and after conjugation and (C) $\Delta_{\mathrm{LSPR}}$ shift. 


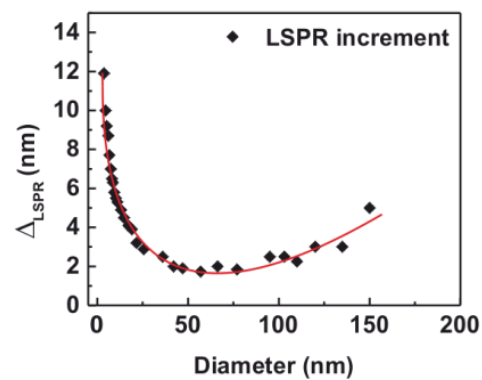

Figure 7. $\Delta_{\text {LSPR }}$ shift of different Au NPs after conjugation with MUA. The whole size range from 3.5 $\mathrm{nm}$ to $200 \mathrm{~nm}$ is covered. Au NPs were synthetized combining the citrate-mediated seeded-growth protocol presented here for the small sizes and the previously published protocol for larger NPs. ${ }^{49}$

\section{CONCLUSIONS}

In the present work, we have shown the possibility to synthesize citrate stabilized Au NPs from 3.5 to $10 \mathrm{~nm}$ by a step by step seeded-growth synthetic protocol based on the reduction of a hydrogen tetrachloroaurate with sodium citrate. The use of traces of tannic acid at the first step, nucleation, allows the formation of $\sim 3.5 \mathrm{~nm}$ Au seeds with narrow size distributions which can be then grown with nanometric resolution. As-synthesized Au NPs possess high uniformity in size, circularity and readily accessible surface. Of special interest are the optical properties since theoretical predictions usually fail for Au NPs smaller than $20 \mathrm{~nm}$ (limit of Mie theory). In this case, additionally to the Localized Surface Plasmon Resonance (LSPR) damping, we observed a size dependence on the LSPR peak position in the range between 3.5 to $10 \mathrm{~nm}$ but interestingly more pronounced when the particles were smaller than $8 \mathrm{~nm}$. Moreover, conjugation experiments with 11-mercaptoundecanoic acid, a model thiol-terminated molecule widely used for Au NP self-assembled monolayer functionalization, further showed that these small particles are also more sensitive to surface modifications.

\section{SUPPORTING INFORMATION}

Details of experimental protocols and conditions studied together with extended Au NPs characterization are available free of charge.

The authors declare no competing financial interest. 


\section{ACKNOWLEDGMENTS}

We acknowledge financial support from the Spanish Ministerio de Ciencia e Innovación (MICINN) (MAT2012-33330) and from the Catalan Agència de Gestió d'Ajuts Universitaris i de Recerca (AGAUR) (2014-SGR-612). Financial support from the QualityNano (INFRA-2010-262163) and FutureNanoNeeds (FP7-NMP-2013-LARGE-7) Projects financed by the European Community under the FP7 Capacities Programme are gratefully acknowledged. N.G.B. acknowledges financial support by MINECO through the Ramon y Cajal program (RYC-2012- 10991) and by the European Commission Seventh Framework Programme (FP7) through the Marie Curie Career Integration Grant (322153-MINE). 


\section{REFERENCES}

(1) Hvolbaek, B.; Janssens, T. V. W.; Clausen, B. S.; Falsig, H.; Christensen, C. H.; Norskov, J. K., Catalytic activity of Au nanoparticles. Nano Today 2007, 2, 14-18.

(2) Homberger, M.; Simon, U., On the application potential of gold nanoparticles in nanoelectronics and biomedicine. Philos. Trans. R. Soc., A 2010, 368, 1405-1453.

(3) Sperling, R. A.; Rivera Gil, P.; Zhang, F.; Zanella, M.; Parak, W. J., Biological applications of gold nanoparticles. Chem. Soc. Rev 2008, 37, 1896-1908.

(4) Tomalia, D. A., In quest of a systematic framework for unifying and defining nanoscience. $J$. Nanopart. Res. 2009, 11, 1251-1310.

(5) Buffat, P.; Borel, J. P., Size effect on the melting temperature of gold particles. Phys. Rev. A 1976, 13, 2287-2298.

(6) Wang, D.; Nap, R. J.; Lagzi, I.; Kowalczyk, B.; Han, S.; Grzybowski, B. A.; Szleifer, I., How and Why Nanoparticle's Curvature Regulates the Apparent pK(a) of the Coating Ligands. J. Am. Chem. Soc. 2011, 133, 2192-2197.

(7) Berciaud, S. p.; Cognet, L.; Tamarat, P.; Lounis, B., Observation of Intrinsic Size Effects in the Optical Response of Individual Gold Nanoparticles. Nano Lett. 2005, 5, 515-518.

(8) Mei, B. C.; Oh, E.; Susumu, K.; Farrell, D.; Mountziaris, T. J.; Mattoussi, H., Effects of Ligand Coordination Number and Surface Curvature on the Stability of Gold Nanoparticles in Aqueous Solutions. Langmuir 2009, 25, 10604-10611.

(9) Comenge, J.; Sotelo, C.; Romero, F.; Gallego, O.; Barnadas, A.; Garcia-Caballero Parada, T.; Dominguez, F.; Puntes, V. F., Detoxifying Antitumoral Drugs via Nanoconjugation: The Case of Gold Nanoparticles and Cisplatin. Plos One 2012, 7.

(10) Choi, H. S.; Liu, W.; Misra, P.; Tanaka, E.; Zimmer, J. P.; Ipe, B. I.; Bawendi, M. G.; Frangioni, J. V., Renal clearance of quantum dots. Nat. Biotechnol. 2007, 25, 1165-1170.

(11) Vasir, J. K.; Reddy, M. K.; Labhasetwar, V. D., Nanosystems in drug targeting: opportunities and challenges. Curr. Nanosci. 2005, 1, 47-64.

(12) Barua, S.; Mitragotri, S., Challenges associated with penetration of nanoparticles across cell and tissue barriers: A review of current status and future prospects. Nano Today 2014, 9, 223-243.

(13) Brust, M.; Walker, M.; Bethell, D.; Schiffrin, D. J.; Whyman, R., Synthesis of ThiolDerivatized Gold Nanoparticles in a 2-Phase Liquid-Liquid System. J. Chem. Soc., Chem. Commun. 1994, 801-802.

(14) Weare, W. W.; Reed, S. M.; Warner, M. G.; Hutchison, J. E., Improved Synthesis of Small $(\mathrm{dCORE} \approx 1.5 \mathrm{~nm})$ Phosphine-Stabilized Gold Nanoparticles. J. Am. Chem. Soc. 2000, 122, 1289012891.

(15) Schmid, G., The relevance of shape and size of $\mathrm{Au}_{55}$ clusters. Chem. Soc. Rev 2008, 37, 19091930.

(16) Leff, D. V.; Brandt, L.; Heath, J. R., Synthesis and Characterization of Hydrophobic, Organically-Soluble Gold Nanocrystals Functionalized with Primary Amines. Langmuir 1996, 12, 4723-4730.

(17) Song, J.; Kim, D.; Lee, D., Size Control in the Synthesis of 1-6 nm Gold Nanoparticles via Solvent-Controlled Nucleation. Langmuir 2011, 27, 13854-13860.

(18) Brust, M.; Fink, J.; Bethell, D.; Schiffrin, D. J.; Kiely, C., Synthesis and reactions of functionalised gold nanoparticles. J. Chem. Soc., Chem. Commun. 1995, 1655-1656. 
(19) Wuelfing, W. P.; Gross, S. M.; Miles, D. T.; Murray, R. W., Nanometer Gold Clusters Protected by Surface-Bound Monolayers of Thiolated Poly(ethylene glycol) Polymer Electrolyte. $J$. Am. Chem. Soc. 1998, 120, 12696-12697.

(20) Kanaras, A. G.; Kamounah, F. S.; Schaumburg, K.; Kiely, C. J.; Brust, M., Thioalkylated tetraethylene glycol: a new ligand for water soluble monolayer protected gold clusters. Chem. Commun. 2002, 2294-2295.

(21) Oh, E.; Susumu, K.; Goswami, R.; Mattoussi, H., One-Phase Synthesis of Water-Soluble Gold Nanoparticles with Control over Size and Surface Functionalities. Langmuir 2010, 26, 76047613.

(22) Hussain, I.; Graham, S.; Wang, Z.; Tan, B.; Sherrington, D. C.; Rannard, S. P.; Cooper, A. I.; Brust, M., Size-Controlled Synthesis of Near-Monodisperse Gold Nanoparticles in the 1-4 $\mathrm{nm}$ Range Using Polymeric Stabilizers. J. Am. Chem. Soc. 2005, 127, 16398-16399.

(23) Male, K. B.; Li, J.; Bun, C. C.; Ng, S.-C.; Luong, J. H. T., Synthesis and Stability of Fluorescent Gold Nanoparticles by Sodium Borohydride in the Presence of Mono-6-deoxy-6pyridinium- $\beta$-cyclodextrin Chloride. J. Phys. Chem. C 2007, 112, 443-451.

(24) Liu, Y.; Male, K. B.; Bouvrette, P.; Luong, J. H. T., Control of the Size and Distribution of Gold Nanoparticles by Unmodified Cyclodextrins. Chem. Mat. 2003, 15, 4172-4180.

(25) Schaaff, T. G.; Whetten, R. L., Giant Gold-Glutathione Cluster Compounds: Intense Optical Activity in Metal-Based Transitions. J. Phys. Chem. B 2000, 104, 2630-2641.

(26) Yonezawa, T.; Yasui, K.; Kimizuka, N., Controlled Formation of Smaller Gold Nanoparticles by the Use of Four-Chained Disulfide Stabilizer. Langmuir 2000, 17, 271-273.

(27) Reiss, H., The Growth of Uniform Colloidal Dispersions. J. Chem. Phys. 1951, 19, 482-487.

(28) LaMer, V. K.; Dinegar, R. H., Theory, Production and Mechanism of Formation of Monodispersed Hydrosols. J. Am. Chem. Soc. 1950, 72, 4847-4854.

(29) Xia, Y.; Xiong, Y.; Lim, B.; Skrabalak, S. E., Shape-Controlled Synthesis of Metal Nanocrystals: Simple Chemistry Meets Complex Physics? Angew. Chem., Int. Ed. 2009, 48, 60-103.

(30) Jana, N. R.; Gearheart, L.; Murphy, C. J., Seeding Growth for Size Control of 5-40 nm Diameter Gold Nanoparticles. Langmuir 2001, 17, 6782-6786.

(31) Zheng, Y. Q.; Zhong, X. L.; Li, Z. Y.; Xia, Y. N., Successive, Seed-Mediated Growth for the Synthesis of Single-Crystal Gold Nanospheres with Uniform Diameters Controlled in the Range of 5150 nm. Part. Part. Syst. Charact. 2014, 31, 266-273.

(32) Alkilany, A. M.; Murphy, C. J., Toxicity and cellular uptake of gold nanoparticles: what we have learned so far? J. Nanopart. Res. 2010, 12, 2313-2333.

(33) Turkevich, J.; Stevenson, P. C.; Hillier, J., A Study of the Nucleation and Growth Processes in the Synthesis of Colloidal Gold. Discuss. Faraday Soc. 1951, 55-\&.

(34) Frens, G., Controlled Nucleation For Regulation Of Particle-Size In Monodisperse Gold Suspensions. Nature, Phys. Sci. 1973, 241, 20-22.

(35) Bastús, N. G.; Sanchez-Tillo, E.; Pujals, S.; Farrera, C.; Lopez, C.; Giralt, E.; Celada, A.; Lloberas, J.; Puntes, V., Homogeneous Conjugation of Peptides onto Gold Nanoparticles Enhances Macrophage Response. ACS Nano 2009, 3, 1335-1344.

(36) Kogan, M. J.; Bastús, N. G.; Amigo, R.; Grillo-Bosch, D.; Araya, E.; Turiel, A.; Labarta, A.; Giralt, E.; Puntes, V. F., Nanoparticle-mediated Local and Remote Manipulation of Protein Aggregation. Nano Lett. 2006, 6, 110-115.

(37) Ji, X.; Song, X.; Li, J.; Bai, Y.; Yang, W.; Peng, X., Size control of gold nanocrystals in citrate reduction: The third role of citrate. J. Am. Chem. Soc. 2007, 129, 13939-13948. 
(38) Schulz, F.; Homolka, T.; Bastús, N. G.; Puntes, V.; Weller, H.; Vossmeyer, T., Little Adjustments Significantly Improve the Turkevich Synthesis of Gold Nanoparticles. Langmuir 2014.

(39) Brown, K. R.; Walter, D. G.; Natan, M. J., Seeding of Colloidal Au Nanoparticle Solutions. 2. Improved Control of Particle Size and Shape. Chem. Mat. 1999, 12, 306-313.

(40) Brown, K. R.; Lyon, L. A.; Fox, A. P.; Reiss, B. D.; Natan, M. J., Hydroxylamine Seeding of Colloidal Au Nanoparticles. 3. Controlled Formation of Conductive Au Films. Chem. Mat. 1999, 12, 314-323.

(41) Brown, K. R.; Natan, M. J., Hydroxylamine Seeding of Colloidal Au Nanoparticles in Solution and on Surfaces. Langmuir 1998, 14, 726-728.

(42) Perrault, S. D.; Chan, W. C. W., Synthesis and Surface Modification of Highly Monodispersed, Spherical Gold Nanoparticles of 50-200 nm. J. Am. Chem. Soc. 2009, 131, 1704217043.

(43) Ziegler, C.; Eychmüller, A., Seeded Growth Synthesis of Uniform Gold Nanoparticles with Diameters of 15-300 nm. J. Phys. Chem. C 2011, 115, 4502-4506.

(44) Niu, J.; Zhu, T.; Liu, Z., One-step seed-mediated growth of 30-150 nm quasispherical gold nanoparticles with 2-mercaptosuccinic acid as a new reducing agent. Nanotechnol. 2007, 18, 325607.

(45) Ojea-Jimenez, I.; Romero, F. M.; Bastus, N. G.; Puntes, V., Small Gold Nanoparticles Synthesized with Sodium Citrate and Heavy Water: Insights into the Reaction Mechanism. J. Phys. Chem. C 2010, 114, 1800-1804.

(46) Ojea-Jimenez, I.; Bastus, N. G.; Puntes, V., Influence of the Sequence of the Reagents Addition in the Citrate-Mediated Synthesis of Gold Nanoparticles. J. Phys. Chem. C 2011, 115, 15752-15757.

(47) Sivaraman, S. K.; Kumar, S.; Santhanam, V., Monodisperse sub-10 nm gold nanoparticles by reversing the order of addition in Turkevich method - The role of chloroauric acid. J. Colloid Interf. Sci. 2011, 361, 543-547.

(48) Rodríguez-Fernández, J.; Pérez-Juste, J.; García de Abajo, F. J.; Liz-Marzán, L. M., Seeded Growth of Submicron Au Colloids with Quadrupole Plasmon Resonance Modes. Langmuir 2006, 22, 7007-7010.

(49) Bastús, N. G.; Comenge, J.; Puntes, V. F., Kinetically Controlled Seeded Growth Synthesis of Citrate-Stabilized Gold Nanoparticles of up to $200 \mathrm{~nm}$ : Size Focusing versus Ostwald Ripening. Langmuir 2011, 27, 11098-11105.

(50) Bastús, N. G.; Merkoçi, F.; Piella, J.; Puntes, V., Synthesis of Highly Monodisperse CitrateStabilized Silver Nanoparticles of up to $200 \mathrm{~nm}$ : Kinetic Control and Catalytic Properties. Chem. Mat. 2014, 26, 2836-2846.

(51) Ahmad, T., Reviewing the Tannic Acid Mediated Synthesis of Metal Nanoparticles. $J$. Nanotechnol. 2014, 2014, 11.

(52) Mühlpfordt, H., The preparation of colloidal gold particles using tannic acid as an additional reducing agent. Experientia 1982, 38, 1127-1128.

(53) Slot, J. W.; Geuze, H. J., A new method of preparing gold probes for multiple-labeling cytochemistry. Eur. J. Cell Biol. 1985, 38, 87-93.

(54) Haiss, W.; Thanh, N. T. K.; Aveyard, J.; Fernig, D. G., Determination of Size and Concentration of Gold Nanoparticles from UV-Vis Spectra. Anal. Chem. 2007, 79, 4215-4221.

(55) Hendel, T.; Wuithschick, M.; Kettemann, F.; Birnbaum, A.; Rademann, K.; Polte, J., In Situ Determination of Colloidal Gold Concentrations with UV-Vis Spectroscopy: Limitations and Perspectives. Anal. Chem. 2014, 86, 11115-11124. 
(56) Lim, S. I.; Ojea-Jiménez, I.; Varon, M.; Casals, E.; Arbiol, J.; Puntes, V., Synthesis of Platinum Cubes, Polypods, Cuboctahedrons, and Raspberries Assisted by Cobalt Nanocrystals. Nano Lett. 2010, 10, 964-973.

(57) Lim, S. I.; Varon, M.; Ojea-Jimenez, I.; Arbiol, J.; Puntes, V., Exploring the Limitations of the Use of Competing Reducers to Control the Morphology and Composition of $\mathrm{Pt}$ and PtCo Nanocrystals. Chem. Mater. 2010, 22, 4495-4504.

(58) Peng, S.; McMahon, J. M.; Schatz, G. C.; Gray, S. K.; Sun, Y., Reversing the sizedependence of surface plasmon resonances. PNAS 2010, 107, 14530-14534.

(59) Link, S.; El-Sayed, M. A., Size and Temperature Dependence of the Plasmon Absorption of Colloidal Gold Nanoparticles. J. Phys. Chem. B 1999, 103, 4212-4217.

(60) Kreibig, U.; Vollmer, M., Optical Properties of Metal Clusters. 1995.

(61) Schulz, F.; Vossmeyer, T.; Bastus, N. G.; Weller, H., Effect of the Spacer Structure on the Stability of Gold Nanoparticles Functionalized with Monodentate Thiolated Poly(ethylene glycol) Ligands. Langmuir 2013, 29, 9897-9908.

(62) Bastús, N. G.; Piella, J.; Puntes, V., Quantifying the Sensitivity of Multipolar (Dipolar, Quadrupolar, and Octapolar) Surface Plasmon Resonances in Silver Nanoparticles: The Effect of Size, Composition, and Surface Coating. Langmuir 2016, 32. 


\section{MATERIALS and METHODS}

Materials. Tetrachloroauric (III) acid ( $\geq 99 \%$ trace metal basis), trisodium citrate dihydrate ( $\geq 99 \%)$, citric acid monohydrate $(\geq 99 \%)$, potassium carbonate $(\geq 99 \%)$ and tannic acid (MW 1701, ACS reagent) were purchased from Sigma-Aldrich. 11-Mercaptoundecanoic acid (95\%) was from Aldrich. Ultrapure water (18.2 M $\Omega$ ) was used for all procedures.

Synthesis of $3.5 \mathrm{~nm}$ seeds Au NPs. A $150 \mathrm{~mL}$ of freshly prepared reducing solution of sodium citrate (SC, $2.2 \mathrm{mM}$ ) containing $0.1 \mathrm{~mL}$ of tannic acid (TA, $2.5 \mathrm{mM}$ ) and $1 \mathrm{~mL}$ of potassium carbonate $\left(\mathrm{K}_{2} \mathrm{CO}_{3}, 150 \mathrm{mM}\right)$ was heated with a heating mantle in a $250 \mathrm{~mL}$ three-necked round-bottom flask under vigorous stirring. When the temperature reached $70{ }^{\circ} \mathrm{C}, 1 \mathrm{~mL}$ of tetrachloroauric acid $\left(\mathrm{HAuCl}_{3}\right.$, $25 \mathrm{mM}$ ) was injected. The color of the solution changed rapidly to black-grey (less than $10 \mathrm{~s}$ ) and then to orange-red in the following 1-2 minutes. The solution was kept at $70{ }^{\circ} \mathrm{C}$ for 5 minutes more to ensure complete reaction of the gold precursor. The resultant particles $\left(\sim 3.5 \mathrm{~nm}, 7 \cdot 10^{13} \mathrm{NPs} / \mathrm{mL}\right)$ were narrowly dispersed, negatively charged and stable for weeks. The addition of $1 \mathrm{mM}$ of $\mathrm{K}_{2} \mathrm{CO}_{3}$ in the reducing solution resulted in a $\mathrm{pH} \sim 10$ which decreased in the reaction mixture to $\mathrm{pH} \sim 8$ because of the addition of $\mathrm{HAuCl}_{4}$. This slightly basic value seems to have an advantageous effect resulting in narrower size distributions of the Au NPs. Other tested conditions from the modification of one of the reaction conditions are described in the supporting information.

Seeded-Growth of Au NPs. Au NPs used as seeds $\left(7 \cdot 10^{13} \mathrm{NPs} / \mathrm{mL}\right)$ were synthetized following above described protocol. Immediately after the synthesis and in the reaction same vessel, the sample was diluted by extracting $55 \mathrm{~mL}$ and adding $55 \mathrm{~mL}$ of SC $(2.2 \mathrm{mM})$. When the temperature reached again $70{ }^{\circ} \mathrm{C}$, two injections of $0.5 \mathrm{~mL}$ of $\mathrm{HAuCl}_{4}(25 \mathrm{mM})$ on a time interval of 10 minutes were done. This growing step comprising sample dilution plus 2 injections of $\mathrm{HAuCl}_{4}$ was repeated until the particles reached the desired size (see Scheme S2).

Conjugation with 11-mercaptoundecanoic acid (MUA). To $1 \mathrm{~mL}$ of as synthetized Au NPs, excess of a basic solution of MUA $(0.1 \mathrm{~mL}, 1 \mathrm{mM})$ was added and stirred gently overnight. Unbounded ligand was removed by further purification of the Au NPs using a regenerated cellulose centrifugal filter (MWCO 10000, Amicon) for particles smaller than $8 \mathrm{~nm}$ or by centrifugation $(15000 \mathrm{~g}, 15$ $\min$.) for particles bigger than $8 \mathrm{~nm}$.

Characterization: Transmission Electron Microscopy (TEM). Au NPs previously conjugated with MUA were visualized using TEM (Jeol 1010) and HRTEM (Tecnai F20 S/TEM). $10 \mu \mathrm{L}$ of the solutions were drop-casted onto a carbon-coated grid and left to dry for at least $24 \mathrm{~h}$. Average size and size distribution of the samples were measured using ImageJ software by counting at least 500 particles. Conjugation/passivation of the Au NP surface with MUA previous to preparation of the grid was a prerequisite to get good TEM images, otherwise close particles had the tendency to coalesce 
once deposited onto the film and under the beam irradiation. UV-vis spectroscopy. UV-Visible spectra were acquired with a Shimadzu UV-2100 using $1.5 \mathrm{~mL}$ plastic cuvettes in the range from $300 \mathrm{~nm}$ to $800 \mathrm{~nm}$. Dynamic Light Scattering. Malvern ZetaSizer Nano ZS (Malvern instruments, UK) operating at a light source wavelength of $532 \mathrm{~nm}$ and fixed scattering angle of $173^{\circ}$ was used to measure $\mathrm{Au}$ NPs hydrodynamic size. Measurements were conducted in $1 \mathrm{~cm}$ path cell and $25^{\circ} \mathrm{C}$ and the samples were previously filtered with $0.2 \mu \mathrm{m}$ regenerated cellulose filter. 
TABLE OF CONTENTS
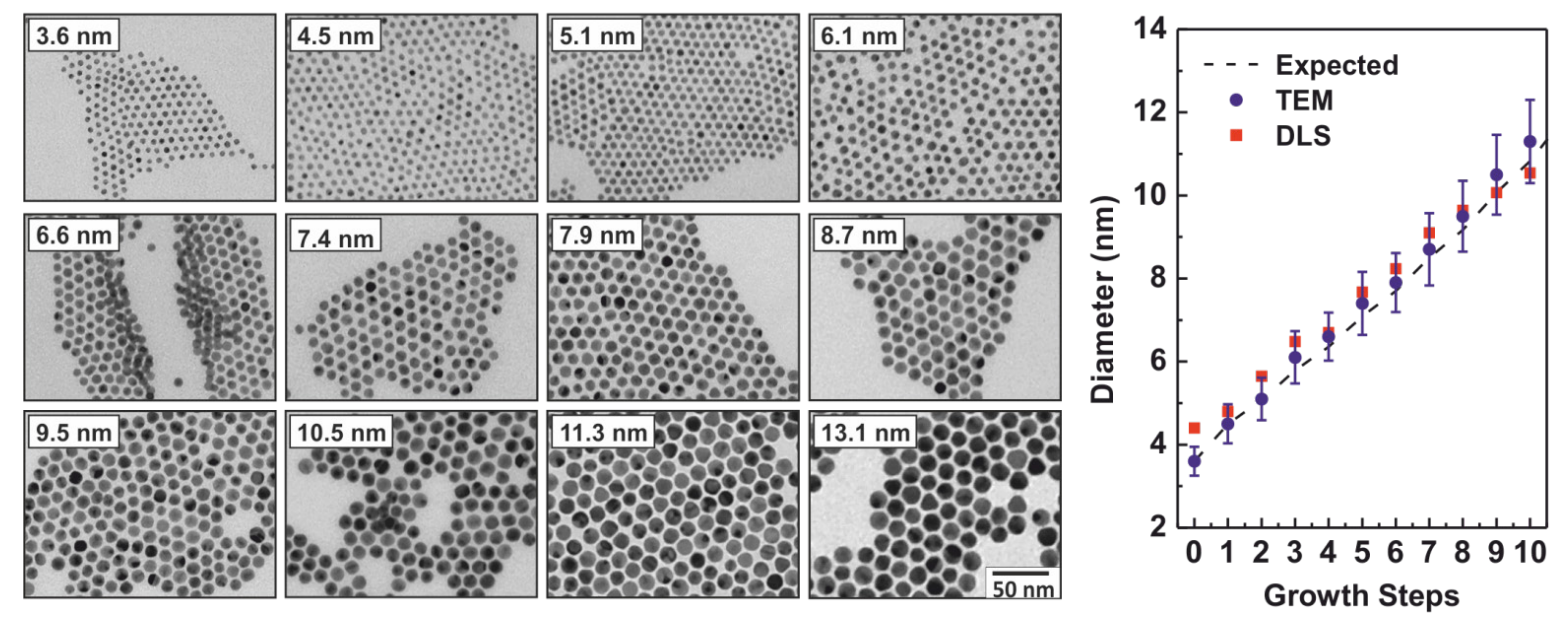